\title{
Use of colchicine in atherosclerotic heart disease
}

\author{
Billy Lin $B A^{1}$, Michael Pillinger $M D^{2}$, Binita Shah $M D^{3}$, Craig Tenner $M D^{1}$
}

Lin B, Pillinger M, Shah B, et al. Use of colchicine in atherosclerotic heart disease. Curr Res Integr Med 2018; 3(S1):2-4.

D espite many recent advances in prevention and treatment, cardiovascular disease remains the leading cause of death worldwide (1). The traditional paradigm of lipid accumulation and the formation of atherosclerotic plaque is increasingly recognized as incomplete, and inadequate to fully explain the underlying mechanisms of cardiovascular risk. Inflammation is now appreciated as a major contributing factor to the progression of atherosclerotic heart disease (ASHD); however, traditional anti-inflammatory treatment with non-steroidal anti-inflammatory drugs (NSAIDs) and glucocorticoids has not only failed to show benefit but appears deleterious (2). The deleterious effects of these medications may not be related to their antiinflammatory actions, but to other unrelated physiological effects that these drugs possess (e.g., hypertension and renal disease for NSAIDs, hypertension and lipid abnormalities for glucocorticoids). Alternatively, the adverse side effect profile of some of these agents may relate to their anti-inflammatory potency in specific settings, such as post-acute myocardial infarction (MI), where some degree of an inflammatory response may be needed for the healing process. Therefore, it may be necessary to focus on anti-inflammatory agents with a more focused target of action and limited or no adverse side effects. The possibility that the benefits of statin therapy on cardiovascular risk may be due, in part, to an anti-inflammatory mechanism supports such a paradigm $(3,4)$. Furthermore, the recently reported Canakinumab Anti-inflammatory Thrombosis Outcomes Study (CANTOS) trial showed a reduction in major adverse cardiovascular events with anti-inflammatory therapy targeting IL-1 in patients post-MI. Unlike with statin therapy, the cardiovascular benefit observed with anti- IL-10 therapy was independent of lipid-lowering therapy and underlines the importance of anti-inflammatory strategies for cardiovascular prevention. Unfortunately, not everyone achieved a significant anti-inflammatory response with canakinumab, and in patients without a significant decrease in high sensitive $\mathrm{C}$-reactive protein (hsCRP) concentration there was no observed cardiovascular benefit (5). Moreover, canakinumab resulted in an increased rate of fatal infections, and the cost of canakinumab is several hundred thousand dollars a year, making this approach impractical for a large population (6).

Colchicine is an ancient medication, currently FDA approved for the treatment of gout and familial Mediterranean fever (7). Although its uses in other rheumatic diseases (e.g., calcium pyrophosphate crystal disease, small vessel vasculitis, Behcet's syndrome) are fairly well established, the potential of colchicine use in ASHD has only recently begun to be studied (8).

Colchicine has multiple mechanisms of action that reduce inflammation, but the mechanisms of benefit that may lead to a decrease in major adverse cardiovascular events in patients with ASHD remain incompletely elucidated. Incorporation of colchicine-bound free tubulin dimers into microtubules blocks subsequent polymerization, which then inhibits leukocyte functions such as superoxide and cytokine release and cell adhesion and migration (9-11). Colchicine can further reduce leukocyte recruitment to inflamed or injured vascular endothelium by modulation of adhesion molecules on the surface of leukocytes and endothelial cells, chemoattractant secretion, and leukocyte deformability (12-14). Colchicine also inhibits the NLRP3 inflammasome and subsequent formation of IL-10, thus suggesting that at least some effects of colchicine may mimic those of canakinumab $(15,16)$. Our group has also shown colchicine to decrease the aggregation between leukocytes and platelets, but not platelet-to-platelet aggregation, suggesting that colchicine may exert an anti-thrombotic mechanism at the site of inflammation without potentially increasing the risk of bleeding (17). In vivo, colchicine has been shown to reduce hsCRP concentration, in patients with ASHD already on statin and anti-platelet therapy (18). Colchicine has also been shown to reduce low attenuation plaque volume on coronary computed tomography angiography, a measure of plaque instability (19). Any or all of these functions are potential mechanisms by which colchicine may reduce cardiovascular events, making it a medication worthy of consideration (20).

The extent of colchicine's effects on clinical cardiovascular outcomes is under study. Our group conducted a retrospective cross-sectional study of 1288 patients with gout, a cohort at high cardiovascular risk, and first reported that treatment with colchicine was associated with a $54 \%$ relative risk reduction in $\mathrm{MI}(1.2 \%$ vs $2.6 \%$; RR 0.46; $\mathrm{p}=0.03)$ (21). A subsequent retrospective cohort study by an independent group examined 501 gout patients and observed a similar protective effect with colchicine, including a $49 \%$ relative risk reduction in a composite outcome of MI, stroke, and transient ischemic attack (22.7 vs. 43.4 events per 1000 patient years; HR $0.5,95 \% \mathrm{CI} 0.30-0.88 ; \mathrm{p}=0.016)$, and a $73 \%$ relative risk reduction in allcause mortality (52.6 vs. 95.1 events per 1000 patient years; HR $0.55,95 \%$ CI $0.35-0.85 ; \mathrm{p}=0.007)(22)$. A single-center, open-label study randomized 532 patients with stable coronary artery disease already on statin and antiplatelet therapy to either colchicine $0.5 \mathrm{mg} /$ day or no colchicine. Low-dose colchicine use resulted in a decrease in cardiovascular events defined as a composite of acute coronary syndromes, out-of-hospital cardiac arrests, and non-cardioembolic ischemic strokes over a median follow-up period of 3 years $(5.3 \%$ vs. $16.0 \%$; HR 0.33 , 95\% CI 0.18-0.59; p<0.001) (23). A Cochrane systematic review of 4992 patients in 39 randomized controlled trials of any clinical setting involving long-term colchicine versus any control demonstrated a significant decrease in $\mathrm{MI}(1.2 \%$ vs. $5.8 \%$; RR $0.2,95 \%$ CI $0.07-0.57 ; \mathrm{p}=0.003)$, but no significant reduction in all-cause mortality (17.8\% vs. $19.3 \%$; RR 0.94, $95 \%$ CI 0.82-1.09; $\mathrm{p}=0.43)$ (24).

The potential of colchicine to reduce angioplasty and stent-related recurrent events has also been evaluated. A randomized, double-blinded study of 196 patients with diabetes mellitus who underwent percutaneous coronary intervention with a bare metal stent demonstrated a reduction in in-stent restenosis as assessed by follow-up angiography $(16 \%$ vs. $33 \%, p=0.007)$ and intravascular ultrasound $(24 \%$ vs. $43 \%, p=0.006)$ with 6 months of twice daily colchicine $(0.5 \mathrm{mg})$ versus placebo $(25)$. However, another doubleblinded, placebo-controlled study of 145 patients undergoing elective coronary angioplasty of 393 lesions without stent placement demonstrated no improvement in the rate of angiographic restenosis with up to 6 months of twice daily colchicine $(0.5 \mathrm{mg})(26)$.

Finally, several studies have assessed the potential role of colchicine specifically in the acute setting. One such study randomized 151 patients ST-segment elevation MI (STEMI) to receive either colchicine ( $2 \mathrm{mg}$ load followed by $0.5 \mathrm{mg}$ twice a day for five days) or placebo and demonstrated a significantly lower infarct size, as measured by CK-MB concentrations, with colchicine use (3144 vs. $6184 \mathrm{ng} / \mathrm{hr} / \mathrm{mL}, \mathrm{p}<0.001)$. A subset of these patients $(\mathrm{n}=60)$ also underwent cardiac magnetic resonance imaging, which confirmed that

${ }^{1}$ Division of General Internal Medicine, Department of Medicine, VA New York Harbor Healthcare System $\mathcal{E}$ New York University School of Medicine, New York, USA; ${ }^{2}$ Division of Rheumatology, Department of Medicine, VA New York Harbor Healthcare System $\mathcal{E}$ New York University School of Medicine, New York, USA; ${ }^{3}$ Division of Cardiology, Department of Medicine, VA New York Harbor Healthcare System $\mathcal{E}$ New York University School of Medicine, New York, USA

Correspondence: Dr Craig Tenner, MD, Division of General Internal Medicine, Department of Medicine, VA New York Harbor Healthcare System $\mathcal{E}$ New York University School of Medicine, New York, USA, Telephone 212-951-5973, e-mail craig.tenner@va.gov k

Received: January 4, 2018, Accepted: January 21, 2018, Published: February 05, 2018

OPEN $\bigcirc$ Access

This open-access article is distributed under the terms of the Creative Commons Attribution Non-Commercial License (CC BY-NC) (http:// creativecommons.org/licenses/by-nc/4.0/), which permits reuse, distribution and reproduction of the article, provided that the original work is properly cited and the reuse is restricted to noncommercial purposes. For commercial reuse, contact reprints@pulsus.com 
colchicine administration resulted in a reduction in infarct size proportional to left ventricular myocardial volume ( $13 \%$ vs. $19.8 \%, p=0.034)(27)$. Our group is currently conducting a single-center, double-blind study in which 400 patients undergoing percutaneous coronary intervention are randomized to either a pre-procedural load of colchicine $(1.2 \mathrm{mg}$ followed by $0.6 \mathrm{mg}$ one hour later) or placebo, and assessed for peri-procedural myocardial necrosis (primary outcomes) and rate of 30-day major adverse cardiovascular events (secondary outcome) (28). A substudy of patients also have a concomitant analysis of inflammatory profiles (co-primary endpoints include neutrophil surface expression of L-selectin and soluble IL-6 concentration) (29). The multicenter, randomized, double-blind Colchicine Cardiovasclar Outcomes Trial (COLCOT) is investigating the effect of long-term administration of colchicine $0.5 \mathrm{mg}$ daily in 4500 patients with a documented MI in the prior 30 days. The primary endpoint is a composite of cardiovascular death, resuscitated cardiac arrest, acute MI, stroke, or hospitalization for angina requiring coronary revascularization (30). The multicenter, international, randomized, double-blind Colchicine and Spironolactone in Patients With STEMI/SYNERGY Stent Registry (CLEAR-SYNERGY) trial is investigating the effects of colchicine $0.5 \mathrm{mg}$ twice a day and/or spironolactone $25 \mathrm{mg}$ once a day following primary percutaneous coronary intervention in the setting of STEMI. The primary outcome measure in the colchicine comparison is a composite of cardiovascular death, recurrent MI, or stroke (31).

Is it time for colchicine to be considered an accepted therapy for cardiovascular treatment and prevention? Probably not quite. First, studies to date have been relatively small, and the data accrued would not likely meet the standard of proof if colchicine were a new drug coming up for first approval. However, there is sufficient mechanistic and pilot clinical data to encourage the careful evaluation of data from the large ongoing trials. Second, while colchicine is generally safe and well-tolerated when prescribed by experienced practitioners, colchicine toxicity is a well-recognized phenomenon and physician education would be needed to ensure that the drug is used safely. Caution with dose-adjustment in patients with renal disease and management of potential drug-drug interactions (particularly with P-glycoprotein and inhibitors, including some statins) requires attentiveness on the part of caregivers (7). Colchicine has a low median lethal dose (LD50), and, therefore, overdose needs to be guarded against carefully. Finally, the appropriate dose and duration of colchicine for cardiovascular indications has yet to be determined: is colchicine's potential benefit likely to come from long-term use or will it be most beneficial during acute events?

Nonetheless, colchicine has a number of advantages that might make it desirable as an add-on therapy to current treatment. First, when used properly, colchicine's kinetics make it relatively easy to comply with once or twice daily dosing. Second, the fact that colchicine has minimal or no independent impact on platelets at the recommended doses makes it a potentially desirable partner to current cardiovascular regiments that typically include multiple drugs that raise the risk of bleeding. Third, while colchicine in the United States is now much more expensive than it was previously, it is still much cheaper than newer agents and biologics-and even cheaper in many other parts of the world. In sum, we seem to be approaching the day when colchicine may be added to the armamentarium for the prevention of cardiovascular disease. Whether and when that day comes will depend on the results of the next round or two of clinical investigation.

\section{REFERENCES}

1. WHO. The top 10 causes of death worldwide. Fact Sheets 2017 January.

2. Bally M, Dendukuri N, Rich B, et al. Risk of acute myocardial infarction with NSAIDs in real world use: Bayesian meta-analysis of individual patient data. Br Med J. 2017;357:j1909.

3. Abeles AM and Pillinger MH. Statins as antiinflammatory and immunomodulatory agents: A future in rheumatologic therapy? Arthritis Rheum. 2006;54(2):393-407.

4. Patti G, Chello M, Pasceri V, et al. Protection from procedural myocardial injury by atorvastatin is associated with lower levels of adhesion molecules after percutaneous coronary intervention: Results from the ARMYDA-CAMs (Atorvastatin for Reduction of MYocardial Damage during Angioplasty-Cell Adhesion Molecules) substudy. J Am Coll Cardiol. 2006;48(8):1560-6.

5. Ridker PM, MacFadyen JG, Everett BM, et al. Relationship of C-reactive protein reduction to cardiovascular event reduction following treatment with canakinumab: A secondary analysis from the CANTOS randomised controlled trial. The Lancet. 2018;391(10118):319-8.
6. Ridker PM, Everett BM, Thuren T, et al. Antiinflammatory therapy with canakinumab for atherosclerotic disease. $N$ Engl J Med. 2017;377(12):1119-31.

7. Administration, U.S.F.a.D. Drug safety information for healthcare professionals - Information for Healthcare Professionals: New Safety Information for Colchicine (marketed as Colcrys). 2017 Nov 26.

8. Slobodnick A, Shah B, Krasnokutsky S, et al. Update on Colchicine. Rheumatology. 2017;57:i2-i11.

9. Andreu JM and Timasheff SN. Tubulin bound to colchicine forms polymers different from microtubules. Proc Natl Acad Sci. 1982;79:6753-6.

10. Dalbeth N, Lauterio TJ, Wolfe HR. Mechanism of action of colchicine in the treatment of gout. Clin Ther. 2014;36(10):1465-79.

11. Chia EW, Grainger R, Harper JL. Colchicine suppresses neutrophil superoxide production in a murine model of gouty arthritis: A rationale for use of low-dose colchicine. Br J Pharmacol. 2008;153(6):1288-95.

12. Cronstein, B.N., et al., Colchicine alters the quantitative and qualitative display of selectins on endothelial cells and neutrophils. J Clin Invest, 1995. 96(2): p. 994-1002.

13. Paschke S, Weidner AF, Paust T, et al. Technical advance: Inhibition of neutrophil chemotaxis by colchicine is modulated through viscoelastic properties of subcellular compartments. J Leukoc Biol. 2013;94(5):1091-6.

14. Li Z, Davis GS, Mohr C, et al. Inhibition of LPS-Induced Tumor Necrosis Factor- $\alpha$ Production by Colchicine and Other Microtubule Disrupting Drugs. Immunobiology. 1996;195(4-5):624-639.

15. Robertson S, Martínez GJ, Payet CA, et al. Colchicine therapy in acute coronary syndrome patients acts on caspase-1 to suppress NLRP3 inflammasome monocyte activation. Clin Sci (Lond). 2016;130(14):1236-46.

16. Leung YY, Yao HLL, Kraus VB. Colchicine--Update on mechanisms of action and therapeutic uses. Semin Arthritis Rheum. 2015;45(3):341-50.

17. Shah B, Allen N, Harchandani B, et al. Effect of colchicine on plateletplatelet and platelet-leukocyte interactions: A pilot study in healthy subjects. Inflammation. 2016;39(1):182-9.

18. Nidorf $\mathrm{M}$ and Thompson PL. Effect of colchicine $(0.5 \mathrm{mg}$ twice daily) on high-sensitivity C-reactive protein independent of aspirin and atorvastatin in patients with stable coronary artery disease. Am J Cardiol. 2007;99(6):805-7.

19. Vaidya K, Arnott C, Martínez GJ, et al. Colchicine therapy and plaque stabilization in patients with acute coronary syndrome: A CT coronary angiography study. JACC Cardiovasc Imaging. 2017.

20. Shah PK. Inflammation and plaque vulnerability. Cardiovasc Drugs Ther. 2009;23(1):31-40.

21. Crittenden DB, Lehmann RA, Schneck L, et al. Colchicine use is associated with decreased prevalence of myocardial infarction in patients with gout. J Rheumatol. 2012;39(7):1458-64.

22. Solomon DH, Liu CC, Kuo IH, et al. Effects of colchicine on risk of cardiovascular events and mortality among patients with gout: A cohort study using electronic medical records linked with Medicare claims. Ann Rheum Dis. 2016;75(9):1674-9.

23. Nidorf SM, Eikelboom JW, Budgeon CA, et al. Low-dose colchicine for secondary prevention of cardiovascular disease. J Am Coll Cardiol. 2013;61(4):404-10.

24. Hemkens LG, Ewald H, Gloy VL, et al. Colchicine for prevention of cardiovascular events. Cochrane Database Syst Rev. 2016(1):CD011047.

25. Deftereos S, Giannopoulos G, Raisakis K, et al. Colchicine treatment for the prevention of bare-metal stent restenosis in diabetic patients. J Am Coll Cardiol. 2013;61(16):1679-85.

26. O'Keefe JH, McCallister BD, Bateman TM, et al. Ineffectiveness of colchicine for the prevention of restenosis after coronary angioplasty. Journal of the American College of Cardiology. 1992;19(7):1597-1600.

27. Deftereos S, Giannopoulos G, Angelidis C, et al. Anti-inflammatory treatment with colchicine in acute myocardial infarction: A pilot study. Circulation. 2015;132(15):1395-403.

28. ClinicalTrials.gov. Colchicine in Percutaneous Coronary Intervention. July 21, 2017 [cited 2017 Dec 10]; Available from: https://clinicaltrials. gov/ct2/show/NCT02594111? cond=NCT02594111\& rank=1. 
29. ClinicalTrials.gov. Anti-inflammatory Effects of Colchicine in PCI. June 26, 2017 [cited 2017 Dec 10]; 3]. Available from: https://clinicaltrials. gov/ct2/show/NCT01709981.

30. ClinicalTrials.gov- Colchicine Cardiovascular Outcomes Tria
(COLCOT) (COLCOT). March 8, 2017 [cited 2017 Dec 10]; Available from: https://clinicaltrials.gov/ct2/show/NCT02551094.

31. ClinicalTrials.gov. Colchicine and Spironolactone in Patients With STEMI / SYNERGY Stent Registry (CLEAR-SYNERGY). October 25, 2017 [cited 2017 Dec 10]; Available from: https://clinicaltrials.gov/ct2/ show/NCT03048825. 